\title{
On universal unfoldings of certain real functions on a Banach space
}

\author{
By R. J. MAGNUS \\ Battelle Research Centre, Geneva
}

(Received 31 March 1976)

The aim of this article is to prove a result which has been thought true for some time. Roughly speaking, if you take a universal unfolding of a germ in finitely many variables, and add to it a non-degenerate quadratic form on an infinite-dimensional space, you still have a universal unfolding.

\section{Notation}

For a pair of Banach spaces $X$ and $Y$, the space of germs at 0 of $C^{\infty}$ mappings from $X$ to $Y$ will be denoted by $\mathscr{E}(X, Y) . \mathscr{E}(X, \mathbb{R})$ will be abbreviated to $\mathscr{E}(X)$. Germs will generally be confused with mappings defined on a neighbourhood of 0 . This is for brevity and to avoid awkward expressions. If $X$ is a Banach space and $X^{*}$ its normed dual, the number $x^{*}(x)$, where $x^{*} \in X^{*}$ and $x \in X$, will be denoted by $\left\langle x^{*}, x\right\rangle$.

LEMma 1. Let $X, A$ be Banach spaces and let $f \in \mathscr{E}(X \times A)$ be such that $f(0, a)=0$ for all $a \in A$. Then $f(x, a)=\langle h(x, a), x\rangle$ where

$$
\begin{aligned}
& h \in \mathscr{E}\left(X \times A, X^{*}\right) . \\
& \text { Proof. } \\
& f(x, a)=\int_{0}^{1} \frac{d}{d t} f(t x, a) d t \\
& =\int_{0}^{1}\left\langle f_{x}(t x, a), x\right\rangle d t \\
& =\left\langle\int_{0}^{1} f_{x}(t x, a) d t, x\right\rangle \text {. }
\end{aligned}
$$

Lemma 2. Let $X$ and $A$ be Banach spaces with $X$ reflexive, let $d \in \mathscr{E}\left(X \times A, X^{*}\right)$ be such that $d(0,0)=0$ and $D_{x} d(0,0)$ is an invertible linear niapping from $X$ onto $X^{*}$. Then for every $f \in \mathscr{E}(X \times A)$, there exist $h \in \mathscr{E}(X \times A, X)$ and $r \in \mathscr{E}(A)$ such that

$$
f(x, a)=\langle d(x, a), h(x, a)\rangle+r(a) .
$$

Proof. The mapping $(x, a) \rightarrow(d(x, a), a)$ is by the inverse function theorem a diffeomorphism of a neighbourhood of $(0,0)$ in $X \times A$ to a neighbourhood of $(0,0)$ in $X^{*} \times A$. Let its inverse be the mapping $\left(x^{*}, a\right) \rightarrow\left(\gamma\left(x^{*}, a\right), a\right)$ where $\gamma \in \mathscr{E}\left(X^{*} \times A, X\right)$. By Lemma 1

$$
f\left(\gamma\left(x^{*}, a\right), a\right)=f(\gamma(0, a), a)+\left\langle x^{*}, k\left(x^{*}, a\right)\right\rangle
$$


where $k \in \mathscr{E}\left(X^{*} \times A, X\right)$ (here we use the identification of $X$ and $\left.X^{* *}\right)$. Now set

and

$$
\begin{aligned}
r(a) & =f(\gamma(0, a), a) \\
h(x, a) & =k(d(x, a), a) .
\end{aligned}
$$

This concludes the proof.

To introduce the main theorem we announce some puzzling terminology. Let $X$ and $A$ be Banach spaces. Then we define $\mathscr{U}(X ; A)$ to be $\mathscr{E}(X \times A)$. Why we should want to do this is given by the next definition. Two members $f$ and $g$ of $\mathscr{U}(X ; A)$ shall be called equivalent if

$$
f(x, a)=g(\phi(x, a), \psi(a))+r(a),
$$

where $\phi \in \mathscr{E}(X \times A, X), \psi \in \mathscr{E}(A, A), r \in \mathscr{E}(A), \phi(\cdot, a)$ is for each $a$, a diffeomorphism on a neighbourhood of $0 \in X, \psi$ is a diffeomorphism on a neighbourhood of $0 \in A$, and finally $\phi(0,0)=0, \psi(0)=0$. Equivalence is an equivalence relation. This definition is slightly different from the usual definition of isomorphism of unfoldings, (see (1)), since $f$ and $g$ need not be unfoldings of the same germ.

Theorem 1. Let $f \in \mathscr{U}(X ; A)$, where $X$ is a reflexive Banach space. Assume that $D_{x} f(0,0)=0$ and $D_{x}^{2} f(0,0)=T$ is a Fredholm operator (since $T$ is symmetric it suffices to assume that it has closed range and finite-dimensional null-space). Let $u_{1}^{*}, \ldots, u_{n}^{*}$ be elements of $X^{*}$ whose projections into $X^{*} / T X$ form a basis of the latter space. Then there is a germ $g \in \mathscr{E}\left(\mathbb{R}^{n} \times A\right)$ such that $f$ is equivalent to a member of $\mathscr{U}(X ; A)$ given by

$$
(x, a) \rightarrow \frac{1}{2}\langle T x, x\rangle+g\left(\left\langle u_{1}^{*}, x\right\rangle, \ldots,\left\langle u_{n}^{*}, x\right\rangle, a\right) .
$$

Suppose further that $\left\{u_{1}, \ldots, u_{n}\right\}$ is a basis of $N(T)$ dual to $\left\{u_{1}^{*}, \ldots, u_{n}^{*}\right\}$. If $D_{x} f(x, 0) \in \operatorname{sp}\left\{u_{1}^{*}, \ldots, u_{n}^{*}\right\}$ whenever $x \in N(T)$ then

$$
g\left(\lambda_{1}, \ldots, \lambda_{n}, 0\right)=f\left(\lambda_{1} u_{1}+\ldots+\lambda_{n} u_{n}, 0\right) .
$$

Proof. Let $Z \subset X$ be the annihilator of $\left\{u_{n}^{*}, \ldots, u_{n}^{*}\right\}$. Every $x$ in $X$ can be written uniquely in the form $z+\sum_{j=1}^{n} \lambda_{j} u_{j}$ where $z \in Z$ and $\lambda_{j}=\left\langle u_{j}^{*}, x\right\rangle$. Furthermore $T$ is an invertible linear mapping of $Z$ onto $T X$, and the latter space is in a natural way the dual of $Z$. Let

$$
F(z, \underline{\lambda}, a)=f\left(z+\Sigma \lambda_{j} u_{j}, a\right)
$$

where $z \in Z, \underline{\lambda}=\left(\lambda_{1}, \ldots, \lambda_{n}\right) \in \mathbb{R}^{n}$ and $a \in A$.

LEMma 3. There exists a $C^{\infty}$ mapping $h$ with range in $Z$ of the variables $z, \underline{\lambda}$, a, and an additional real variable $t$. The domain of $h$ is defined by relations of the form

$$
\max (\|z\|,|\underline{\lambda}|,\|a\|)<\epsilon ; \quad-\epsilon<t<1+\epsilon .
$$

The following equation holds:

$$
F(z, \underline{\lambda}, a)-\frac{1}{2}\langle T z, z\rangle=\left\langle t D_{z} F(z, \underline{\lambda}, a)+(1-t) T z, h(z, \underline{\lambda}, a, t)\right\rangle+\psi(\underline{\lambda}, a, t) .
$$

In this equation $\psi$ is defined on the same domain as $h$ but does not depend on $z$.

Proof of lemma. By Lemma 2 we can define ' $h$ ' and ' $\psi$ ' for $t$ in a neighbourhood of any point $t_{0} \in[0,1]$. Just take $Z$ to be the space $X$ of Lemma 2 and amalgamate the other variables into the $A$ of Lemma 2. Pick a finite number of these neighbourhoods, say $V_{1}, \ldots, V_{k}$, and a partition of unity of $[0,1]$ relative to them, say, $\phi_{1}, \ldots, \phi_{k}$. 
Let the ' $h$ ' and ' $\psi$ ' on $V_{j}$ be denoted by $h_{j}$ and $\psi_{j}$, these mappings being extended by setting them equal to zero for $t \notin V_{j}$. Finally we define $h=\Sigma \phi_{j} h_{j}$ and $\psi=\Sigma \phi_{j} \psi_{j}$. These satisfy the required conditions.

Completion of proof of theorem. Let $w(z, \underline{\lambda}, a, t)$ be the solution of the differential equation

$$
\dot{\zeta}=-h(\zeta, \underline{\lambda}, a, t) \quad(\zeta \in Z)
$$

such that $w(z, \underline{\lambda}, a, 0)=z$. Define

Set

$$
g(\underline{\lambda}, a)=\int_{0}^{1} \psi(\underline{\lambda}, a, t) d t .
$$

$$
M(z, \underline{\lambda}, a, t)=t F^{\prime}(z, \underline{\lambda}, a)+(1-t) \frac{1}{2}\langle T z, z\rangle .
$$

Equation (1) now implies

$$
(d / d t) M(w(z, \underline{\lambda}, a, t), \underline{\lambda}, a, t)=\psi(\underline{\lambda}, a, t) .
$$

Integrating between 0 and 1

$$
F(w(z, \underline{\lambda}, a, 1), \underline{\lambda}, a)=\frac{1}{2}\langle T z, z\rangle+g(\underline{\lambda}, a) .
$$

This implies the required equivalence, since the mapping $z \rightarrow w(z, \underline{\lambda}, a, 1)$ is a diffeomorphism.

A note on the existence of solutions of (2): by considering Lemmas 1 and 2 it may be seen that $h(0,0,0, t) \equiv 0$. Hence the solution of (2) with initial value 0 exists for all $t$ when $\underline{\lambda}=0$ and $a=0$. It is just $\zeta=0$. The set of quadruples $(z, \underline{\lambda}, a, t)$ such that the solution of $(2)$ with initial values $z$ exists on the interval $[0, t]$ is open. Hence this set contains an open neighbourhood of the set $\{(0,0,0, t): 0 \leqslant t \leqslant 1\}$, which is what we require.

To obtain the last part, note that the stated assumption is equivalent to $D_{z} F(0, \underline{\lambda}, 0)=0$. Then by $(1), \psi(\underline{\lambda}, 0, t)=F(0, \underline{\lambda}, 0)=f\left(\Sigma \lambda_{j} u_{j}, 0\right)$, whence the result.

We shall now state two corollaries which use the idea of a universal unfolding. Let $\eta \in \mathscr{E}(X)$ and let $f \in \mathscr{U}(X ; A)$ be such that $f(x, 0)=\eta(x) . f$ is called an unfolding of $\eta$. $f$ is called a universal unfolding of $\eta$ if given $\mathrm{g} \in \mathscr{U}(X ; B)$ such that $g(x, 0)=\eta(x)$, we have

$$
g(x, b)=f(\phi(x, b), \psi(b))+r(b),
$$

where $\phi \in \mathscr{E}(X \times B, X), \psi \in \mathscr{E}(B, A), r \in \mathscr{E}(B), \phi(\cdot, b)$ is, for each $b$, a diffeomorphism of a neighbourhood of $0 \in X$ such that $\phi(\cdot, 0)$ is the identity, and $\psi(0)=0$. Since $\psi$ need not be invertible the relation expressed by (3) is quite different from equivalence. If for some $f \in \mathscr{U}(X ; A)$ and $g \in \mathscr{U}(X ; B)$ the relation (3) holds, except that $\phi(\cdot, 0)$ need not be the identity, we shall say that $g$ is induced from $f$.

Corollary 1. Let $Z$ be a reflexive Banach space, $T: Z \rightarrow Z^{*}$ a symmetric linear homeomorphism. Let $\eta \in \mathscr{E}\left(R^{n}\right)$ such that $\eta^{\prime}(0)=0$ have a universal unfolding $f \in \mathscr{U}\left(\mathbb{R}^{n} ; A\right)$. Then the germ $(z, \underline{\lambda}) \rightarrow \frac{1}{2}\langle T z, z\rangle+\eta(\underline{\lambda})$ which is a member of $\mathscr{E}\left(Z \times \mathbb{R}^{n}\right)$ has $a$ universal unfolding $(z, \underline{\lambda}, a) \rightarrow \frac{1}{2}\langle T z, z\rangle+f(\underline{\lambda}, a)$.

Corollary 2. Let $\eta \in \mathscr{E}(X)$, where $X$ is reflexive, be such that $\eta^{\prime}(0)=0$ and $\eta^{\prime \prime}(0)=T$ is a Fredholm operator. Let $\left\{u_{1}, \ldots, u_{n}\right\}$ be a basis for $N(T)$ and suppose there exists a 
topological supplement $Z$ of $N(T)$ with the property that for any $x \in N(T)$ and $z \in Z$, $\left\langle\eta^{\prime}(x), z\right\rangle=0$. Let $\gamma(\underline{\lambda})=\eta\left(\lambda_{1} u_{1}+\ldots+\lambda_{n} u_{n}\right)$ and let $g(\underline{\lambda}, a)$ be a universal unfolding of $\gamma$. Then any unfolding of $\eta$ is induced from the unfolding germ

where $x=z+\Sigma \lambda_{j} u_{j}, z \in Z$.

$$
(x, a) \rightarrow \frac{1}{2}\langle T z, z\rangle+g(\underline{\lambda}, a)
$$

Example. This is taken from (2). Let $X=\left\{x \in H^{2}[-1,1]: x(-1)=x(1)=0\right\}$. $H^{2}[-1,1]$ is the space of $L^{2}$-functions on $[-1,1]$, whose first- and second-order distribution derivatives are $L^{2}$-functions. Such functions are continuous, and it is a Hilbert space. Define $\eta \in \mathscr{E}(X)$ by

$$
\eta(x)=\frac{1}{2} \int_{-1}^{1}\left(\left|x^{\prime \prime}(s)\right|^{2}-\frac{\pi^{2}}{4}\left|x^{\prime}(s)\right|^{2}\right) d s+\frac{k}{8}\left(\int_{-1}^{1}\left|x^{\prime}(s)\right|^{2} d s\right)^{2},
$$

where $k$ is a constant. The physical meaning of $\eta$ according to (2) is the following. Consider an elastic beam of small cross-section fixed between the points -1 and +1 , and subjected to an increasing compressive stress exactly in line with it. Then at a certain value of the stress the beam buckles. If, with this value of the stress, the shape of the beam happened to be described by the function $x(s)$, then $\eta(x)$ would be its elastic energy. In reality the configuration of the beam is supposed to be a function $x$ such that $\eta^{\prime}(x)=0$.

We have

$$
\begin{aligned}
& \left\langle\eta^{\prime}(x), u\right\rangle=\int_{-1}^{1}\left(x^{\prime \prime}(s) u^{\prime \prime}(s)-\frac{\pi^{2}}{4} x^{\prime}(s) u^{\prime}(s)\right) d s+\frac{k}{2}\left(\int_{-1}^{1}\left|x^{\prime}(s)\right|^{2} d s\right)\left(\int_{-1}^{1} x^{\prime}(s) u^{\prime}(s) d s\right) \\
& \left\langle\eta^{\prime \prime}(0) v, u\right\rangle=\int_{-1}^{1}\left(v^{\prime \prime}(s) u^{\prime \prime}(s)-\frac{\pi^{2}}{4} v^{\prime}(s) u^{\prime}(s)\right) d s .
\end{aligned}
$$

That $T=\eta^{\prime \prime}(0)$ is a Fredholm operator and that its kernel is spanned by $\cos \frac{1}{2} \pi s$ is shown in (2). Now

Hence if we define

$$
\left\langle\eta^{\prime}\left(\lambda \cos \frac{1}{2} \pi s\right), u\right\rangle=\operatorname{const} \lambda^{3} \int_{-1}^{1} u(s) \cos \frac{1}{2} \pi s d s .
$$

$$
Z=\left\{z \in X: \int_{-1}^{1} z(s) \cos \frac{1}{2} \pi s d s=0\right\}
$$

then $Z$ has the properties required in Corollary 2. Finally

$$
\eta\left(\lambda \cos \frac{1}{2} \pi s\right)=\text { const } \lambda^{4} .
$$

The germ $\lambda^{4}$ has a universal unfolding $\left(\lambda, a_{1}, a_{2}\right) \rightarrow \lambda^{4}+a_{1} \lambda^{2}+a_{2} \lambda$, (see (3)). Hence any unfolding of $\eta(x)$ is induced from

$$
\begin{aligned}
x & \rightarrow \lambda^{4}+a_{1} \lambda^{2}+a_{2} \lambda+Q(z), \\
& \lambda=\int_{-1}^{1} x(s) \cos \frac{1}{2} \pi s d s,
\end{aligned}
$$

$z=x-\lambda \cos \frac{1}{2} \pi s$, and $Q$ is a non-degenerate quadratic form on $Z$. 


\section{REFERENCES}

(1) The classification of elementary catastrophes of codimension $\leqslant 5$. Lectures by E. C. Zeeman (Spring 1973). Notes written and revised by D. J. A. Trotman.

(2) Chillingworth, D. The catastrophe of a buckling beam. Proceedings of the Symposium on Applications of Topology and Dynamical Systems, University of Warwick, 1973/4 (ed. A. K. Manning).

(3) Тном, R. Stabilité structurelle et morphogénèse (Benjamin, 1972). 